complaint belongs to 'illness behaviour', which is different from 'objective symptoms' assessed by psychiatrists, preferably using a standardised procedure.

Littlewood mentions the Western patterns of eating disorders, multiple personality disorder, overdosing, shoplifting, agoraphobia and school refusal. Many of these, if not all, are also found in non-Western societies (e.g., see Kleinman \& Lin, 1981). Furthermore, school refusal is not a formal diagnosis in either the ICD-10 or the DSM-IV; rather, it is a behavioural problem possibly with underlying 'etic' psychopathology (depression, separation anxiety, phobia, learning disorders and so forth) and socio-environmental factors. In any society, primitive or modern, there are certain forms of teaching activity not run by modern school institutions. Presumably, the same refusal to attend these various forms of 'school' exists, with similar underlying psychiatric and socio-environmental factors. The ways of this refusal and the context of the socio-environmental factors are likely to be 'emic'. For effective management of school refusal, both the underlying potential etic psychopathology and the emic illness behaviour and socio-environmental factors must be carefully examined. This is an alternative example of what I intended to elaborate using the example of koro.

The long-standing debate over etic/emic and semantic issues in cross-cultural psychiatry is unlikely to be satisfactorily resolved in the near future. However, it is believed that the development of standardised clinical interviews with emphasis on cross-cultural equivalence at the level of symptoms (e.g., Cheng et al, 2001) helps to avoid the so-called "category fallacy" (Kleinman, 1987).

It should be stressed that the underreporting of psychological symptoms by interviewees from developing nations that I mentioned in my editorial does not mean that these people do not have, or cannot differentiate, emotions. People are people, and the very low rate of reporting of psychological symptoms to doctors by people in developing countries may be due to greater social stigma towards mental illness, their lack of knowledge about mental illness and a much less psychologically oriented medical practice. More studies into this area are needed, and I believe that anthropologically oriented researchers can make a great contribution to this endeavour.

The etic/emic approach to psychopathology does not imply that psychiatry is confined only to biology. The emic pathoplastic shaping and illness behaviour closely associated with different sociocultural settings are equally important in psychiatry and require culture-specific approaches in combination with biological treatment. After all, mental disorders are believed to be the product of gene/environment interaction (Cheng \& Cooper, 2001).

Cheng, A.T. A. \& Cooper, B. (eds) (200I) Genome and envirome: their roles and interaction in psychiatric epidemiology. British Journal of Psychiatry, 178 (suppl. 40)

_ , Tien, A. Y., Chang, C. J., et al (2001) Crosscultural implementation of a Chinese version of the Schedule for Clinical Assessment in Neuropsychiatry (SCAN) in Taiwan. British journal of Psychiatry, 178, 567-572.

Kleinman, A. (1987) Anthropology and psychiatry The role of culture in cross-cultural research on illness. British journal of Psychiatry, I5I, 447-454.

— \& Lin, T.Y. (eds) (1981) Normal and Abnorma Behaviour in Chinese Culture. Dordrecht: D. Reidel.

A.T. A. Cheng Institute of Biomedical Sciences, Academia Sinica, Taipei II529, Taiwan

\section{Cross-cultural psychiatric interviews and research instruments}

We read Andrew Cheng's (2001) editorial with much interest. We strongly agree that the development of cross-culturally comparable diagnostic interviews is a pressing need.

In a recent survey in our unit in Sri Lanka of 43 patients presenting with depressive disorder, one-third of these on presentation made a subjective complaint of a "burning sensation of the body" (literal translation) and related secondary distress and denied having most of the core depressive symptoms although the symptom manifestation was of a depressive disorder. Thus, finding semantic or psycholinguistic equivalence for psychiatric symptoms across cultures will be a challenging, albeit necessary, exercise.

We believe that the lack of valid diagnostic tools is an important factor in the limited capacity for psychiatric research in developing countries, which in turn contributes to the underrepresentation of such research in high-impact journals noted by Patel \& Sumathipala (2001)

A case in point is that in Sri Lanka the only validated psychiatric rating scales in the native languages are the Mini-Mental State Examination (MMSE) and the General Health Questionnaire (GHQ-30). Efforts at validating the Hospital Anxiety and Depression (HAD) scale (D. de Silva, personal communication, 2001) in Sinhala (the language of the majority) show that the sensitivity and specificity of such an instrument is low. This is noteworthy considering the fact that locally developed diagnostic instruments may not find ready acceptance in high-impact journals.

Cheng, A. T. A. (200I) Case definition and culture: are people all the same? British Journal of Psychiatry, 179 $1-3$.

Patel, V. \& Sumathipala, A. (200I) International representation in psychiatric literature. Survey of six leading journals. British Journal of Psychiatry, I78, 406-409.

K. A. L. A. Kuruppuarachchi,

S. S. Williams Faculty of Medicine, University of Kelaniya, PO Box 6, Thalagolla Road, Ragama, Sri Lanka

\section{Mental and physical illness}

The editorial by Kendell (2001) independently reflects the view of Baker \& Menken (2001) that it is time to abandon the term 'mental illness'. All three authors emphasise that an important reason for so doing is that the term is stigmatising and undermining of the care and treatment of millions of psychiatric (Kendell) and neurological patients (Baker \& Menken). Interestingly, Kendell suggests that the term 'psychiatric illness' is more acceptable, whereas Baker $\&$ Menken propose instead 'brain illness'. The former seems to replace the mind by the psyche and the latter by the brain.

Like Kendell, I have reviewed the historical processes that have led to the evolution and divergence of psychiatry and neurology as separate disciplines with all the ensuing confusing theoretical and practical uncertainties and complications for professionals and patients alike, including stigma (Reynolds, 1990). Modern neuroscience, which has demonstrated how brain function is profoundly influenced by psychological and social as well as biological factors, has opened the way for resolving some of these uncertainties and divisions. I share the view that one way forward is to build practical bridges between neurology and psychiatry (Reynolds \& Trimble, 1989). For example, it does not make sense for neurologists and psychiatrists quite separately to tackle the problem of stigma towards brain and mental illnesses without 
some dialogue or coordination, especially as neurologists sometimes blame the stigma of brain disease on misunderstandings created by inappropriate application of the term 'mental'.

I do not, however, share the possibly unrealistic and impractical view that the term 'mental illness' should be abandoned. Brain/mind issues have been debated by professionals, philosophers, patients and the public for centuries, and this will continue for some time to come. It is reminiscent of proposals to abolish the word 'epilepsy' because this neurological condition is so stigmatised. Similar suggestions have been made in the past for the words 'cancer' and 'leprosy', which together with 'epilepsy' were three great unmentionables for much of the 20th century (Reynolds, 2000).

Stigma results from ignorance, misunderstanding, fear and prejudice, and the way to combat it is by education and raising public awareness. Rather than abandon the word 'epilepsy' the International League Against Epilepsy (professional), the International Bureau for Epilepsy (patients) public) and the World Health Organization (political) have jointly initiated a global campaign to bring epilepsy 'out of the shadows' (Reynolds, 2000).

Baker, M. \& Menken, M. (200I) Time to abandon the term mental illness. BMJ, 322, 937.

Kendell, R. E. (200I) The distinction between mental and physical illness. British Journal of Psychiatry, $\mathbf{1 7 8}$ 490-493.

Reynolds, E. H. (1990) Structure and function in neurology and psychiatry. British Journal of Psychiatry I57. $481-490$

- (2000) The ILAE/IBE/WHO Global Campaign against Epilepsy: bringing epilepsy "out of the shadows". Epilepsy and Behaviour, I(suppl.), S3-S8.

— \& Trimble, M. R. (eds) (1989) The Bridge between Neurology and Psychiatry. Edinburgh: Churchill Livingstone.

E. H. Reynolds Institute of Epileptology, Weston Education Centre, King's College, Denmark Hill Campus, Cutcombe Road, London SE5 9PJ, UK

Kendell (2001) begins his editorial on the distinction between mental and physical illness by quoting with approval Lady Mary Wortley Montagu's comment that "madness is as much a corporeal distemper as the gout or asthma". This suggests that he might be a physicalist, that is an advocate of the view that all facts about mind and mentality are physical facts, but at no point does he say this explicitly. He is critical of
Cartesian dualism - without saying exactly why.

Kendell then makes a proposal of his own: "In reality, neither minds nor bodies develop illnesses. Only people (or, in a wider context, organisms) do so, and when they do both mind and body, psyche and soma, are usually involved". But he does not explain how the individual person, the mind and the body are supposed to be related to one another and how this would heal the Cartesian split, nor does he offer any arguments in favour of this suggestion. If illnesses can be attributed only to people and not to minds or bodies, then we might expect Kendell to want to talk only of illnesses in general, and not of two different types of illness, as he continues to do in this editorial. Astonishingly, in the very next sentence he appears to be endorsing Cartesian dualism, the view he has already rejected: "Pain, the most characteristic feature of so-called bodily illness, is a purely psychological phenomenon". If pain is a "purely psychological phenomenon", then it can have no physical component. So there is at least one purely psychological, nonphysical phenomenon in the world - a fact that is incompatible with physicalism. But, apparently oblivious of this, Kendell again dismisses Cartesian dualism when he observes that "the differences between mental and physical illnesses... are quantitative rather than qualitative", a remark that suggests physicalism again. Just how could differences between mental and physical illnesses by quantified? How can phenomenal consciousness or 'raw feelings' (i.e. what it is like to have certain mental experiences, such as pain or pleasure, visual hallucinations or paranoid delusions) differ only quantitatively and not qualitatively from physical phenomena?

Kendell seems to teeter between Cartesian dualism and physicalism and he presents no arguments for an alternative to dualism that might lend support to his proposed changes in terminology.

Kendell, R. E. (200I) The distinction between mental and physical illness. British Journal of Psychiatry, I78, 490-493.

P. Crichton Department of Psychologica Medicine, Royal Marsden Hospital, Fulham Road, London SW3 6JJ, UK

Kendell's (2001) editorial made two mistakes in its reasoning, which led to an unhelpful conclusion. One cannot say that mental and physical illness should be conflated because, irrespective of the balance, mental and physical symptoms are expressed in both. This is insisting that differences in degree are not differences at all. There are indeed many disorders that have both mental and physical expressions. However, to claim that anxiety-related chest pain and myocardial infarction are both physical disorders is to conflate precisely what we wish to distinguish, even if anxiety can cause both. We contrast the terms 'mental' and 'physical' because the contrast says what we mean, and we have good reason for meaning it. As Kendell himself points out, no alternative has been found.

Proposing that disturbances in bodily function are necessary for psychiatric disorder does not imply that psychiatric disorders are physical disorders. Consider a computer virus. It may exist as a series of electrical states in a computer, a set of statements in a computer language, even a series of thoughts in someone's head, so its existence is not dependent on any physical object. None the less, it may disrupt a computer's function despite there being no physical fault in the machine. It is generally accepted that such arguments show that mental states might themselves be functions (Heil, 1998), and so purely functional psychiatric disorders are quite possible.

These mistakes lead Kendell to suggest that stigma might be reduced if all psychiatric disorders were to be regarded as physical. This makes mental illness literally unspeakable. But not speaking of something true implies an attitude towards it of denial, shame and horror, not acceptance. The concept of mental health and its promotion is currently competing successfully with 'madness' in popular culture. By falsely declaring 'mental' to be meaningless, the editorial threatens this progress. It may also consign those of our patients who are not sufficiently biological in their pathology to that therapeutic underclass, the 'worried well'.

Heil, J. (1998) Philosophy of Mind: A Contemporary Introduction. New York: Routledge.

Kendell, R. E. (200I) The distinction between mental and physical illness. British Journal of Psychiatry, $\mathbf{1 7 8}$ 490-493.

D. M. Foreman Department of Psychiatry, Keele University, Thornburrow Drive, Hartshill, Stoke-on-Trent ST4 7QB, UK

Author's reply: I agree with much of what Dr Reynolds says and with Baker \& 